\title{
The Pricing of European Exchange Option
}

\author{
Shougang Zhang and Yunfeng Yang* \\ School of Science XI'AN University of Science and Technology, XI'AN 710054, PR China \\ ${ }^{*}$ Corresponding author
}

\begin{abstract}
This paper discusses the problem of pricing on some multi-asset option European exchange option in jump-diffusion model by martingale method. By changing basic assumption of William Margrabe exchange option pricing model to the assumption that jump process is count process that more general than Poisson process, it is established that the behavior model of the stock pricing process is jump-diffusion process. The formula of European exchange option whose stock price with jump process is a count process that more general than Poisson process is deduced under the risk-neutral hypothesis, and it is extended that William Margrabe exchange option pricing model.
\end{abstract} process

Keywords-European exchange options; jump-diffusion; count

\section{INTRODUCTION}

This Throughout the nineties, we have seen the synergistic union of mathematics, finance, the computer, and the global economy. Currency markets trade tow trillion dollars per day, and sophisticated financial derivatives such as options, swaps, and quantors are commonplace. Since the appearance of the Black-Scholes formula in $1973^{[1]}$, the financial community has embraced an abundant and ever-expanding array of mathematical tolls and models. Continuous-time mathematics has become one of the essential tools of modern finance. The elegant mathematics of stochastic calculus simplifies the solution of a wide range of important problems in finance. William Margrabe(1978) ${ }^{[2]}$ study an equation for the value of the option to exchange one risky asset for another. His theory grows out of the brilliant Black-Scholes(1973) solution to the longstanding call option pricing problem---which assumes that the price of a riskless discount bond grew exponentially at the riskless interest rate---and Merton's(1973) ${ }^{[3]}$ extension---in which the discount bond's value is stochastic until maturity. But we show that real data cannot always be fit by a geometric Brownian motion model, and that more general models may need to be considered. The appearance of important information will cause the stock price to a kind of not continual jumps ${ }^{[4-6]}$.A mass of finance practice has indicated that there is a serious warp between the hypothesis of Black-Scholes model about the underlying asset price and the realistic markets. Therefore, many scholars put forward many new kinds of option pricing models by relaxing some assuming conditions of Black-Scholes model. Option pricing theory with jumpdiffusion is one of them. In this paper, I develop an equation for the value of the option to exchange one risky asset for another. Establish the option-pricing model when exercise price is random variable. The option-pricing model is options to exchange one asset to another. Pricing formula of European option is also given.

\section{ASSUMPTIONS AND MODELS}

Let $\left(\Omega, F, P,\left(F_{t}\right)_{0 \leq t \leq T}\right)$ be a probability space and $\left\{W_{t}^{2}, W_{t}^{3}, 0 \leq t \leq T\right\}$ be a two-dimensional standard Wiener process given on a probability space $(\Omega, F, P)$. The market is built with a bond $B_{t}$ and two risky assets $S_{t}^{1}, S_{t}^{2}$. We suppose that $B_{t}$ is the solution of the equation

$$
\frac{d B_{t}}{B_{t}}=r d t ; \quad B_{0}=1 \text {, }
$$

and $S_{t}^{1}, S_{t}^{2}$ satisfies the stochastic differential equation

$$
\begin{aligned}
& \frac{\mathrm{d} S_{t}^{1}}{S_{t^{-}}^{1}}=r \mathrm{~d} t+\sigma_{1} \mathrm{~d} W_{t}^{1}+(V-1) \mathrm{d} \tilde{N}_{t} \\
& \frac{\mathrm{d} S_{t}^{2}}{S_{t^{-}}^{2}}=r \mathrm{~d} t+\sigma_{2} \mathrm{~d} W_{t}^{2}+(U-1) \mathrm{d} \tilde{N}_{t}
\end{aligned}
$$

where risk-free interest rate $r$ and volatility $\sigma_{1}, \sigma_{2}$, are supposed to be constant. $\left\{W_{t}^{1}, 0 \leq t \leq T\right\}$ ( $\left.\left\{W_{t}^{1}=\rho W_{t}^{2}+\sqrt{1-\rho^{2}} W_{t}^{3}, 0 \leq t \leq T\right\}\right), \quad\left\{W_{t}^{2}, 0 \leq t \leq T\right\}$ are standard Wiener process on a suitable probability space $(\Omega, F, P)$. The correlation between the $\left\{W_{t}^{1}, 0 \leq t \leq T\right\}$ and $\left\{W_{t}^{2}, 0 \leq t \leq T\right\}$ is $\rho$, $\left\{\tilde{N}_{t}, 0 \leq t \leq T\right\}$ is the compensated martingale of nonexplosive counting process ${ }^{[7]}\left\{N_{t}, 0 \leq t \leq T\right\}$ with intensity parameter $\lambda(t)$ and $V_{0}, V_{1}, V_{2}, \cdots V_{N_{t}} \quad\left(V_{i}>0, V_{0}=0\right) \quad\left(V_{i}\right.$ is independent of $V_{j}$, for $i \neq j), \quad U_{0}, U_{1}, U_{2}, \cdots U_{N_{t}} \quad\left(U_{i}>0, U_{0}=0\right) \quad\left(U_{i}\right.$ is independent of $U_{j}$, for $i \neq j$ )are the random variable percentage change in the money supply if the count process occurs. The random variables $\left\{W_{t}^{2}, 0 \leq t \leq T\right\}, \quad\left\{W_{t}^{3}, 0 \leq t \leq T\right\}, \quad\left\{N_{t}, 0 \leq t \leq T\right\} \quad$ and $\left\{V_{i}, 1 \leq i \leq N_{t}\right\}, \quad\left\{U_{i}, 1 \leq i \leq N_{t}\right\}$ are assumed to be mutually independent.

We assume that the filtration $\left(F_{t}, 0 \leq t \leq T\right)$ is generated by the martingale $\left\{\tilde{N}_{t}, 0 \leq t \leq T\right\}$ and $\left\{W_{t}^{1}, 0 \leq t \leq T\right\},\left\{W_{t}^{2}, 0 \leq t \leq T\right\}$.

Assumption $1 \mathrm{P}$ is risk-neutral martingale measure ${ }^{[8]}$.

Assumption $2 \sigma_{1}^{2}-2 \sigma_{1} \sigma_{2} \rho+\sigma_{2}^{2} \neq 0$. 
Lemma 1 Let $\frac{d P^{*}}{d P}=\prod_{i=1}^{N_{T}} V_{i} \exp \left\{-\frac{1}{2} \sigma T+\sigma W_{T}+(1-E(V)) \int_{0}^{T} \lambda(s) d s\right\}$, then, under the martingale measure $P^{*}$, process $\left\{N_{t}, 0 \leq t \leq T\right\}$ is nonexplosive counting process with intensity parameter $E(V) \lambda(t)$, and

$$
\begin{gathered}
P_{n}^{*}(T)=P_{n}(T)(E(V))^{n} \exp \left\{(1-E(V)) \int_{0}^{T} \lambda(s) d s\right\} \\
E_{*}\left[f\left(V_{1}, V_{2}, \cdots, V_{n}\right)\right]=\frac{1}{(E(V))^{n}} E\left[f\left(V_{1}, V_{2}, \cdots, V_{n}\right) \cdot \prod_{i=1}^{n} V_{i}\right]
\end{gathered}
$$

If the jumps $V_{i}, i \geq 1$ have a lognormal distribution with mean parameter $\mu_{0}$ and variance $\sigma_{0}^{2}$ under the martingale measure $P$,then $\ln V_{i}, i \geq 1$ have a lognormal distribution with mean parameter $\mu_{0}+\sigma_{0}{ }^{2}$ and variance $\sigma_{0}^{2}$ under the martingale measure $P^{*}$.

\section{Proof}

$$
\begin{aligned}
P_{n}^{*}(T) & =E_{*}\left[I_{\left\{N_{T}=n\right\}}\right]=E\left[\frac{d P^{*}}{d P} I_{\left\{N_{T}=n\right\}}\right] \\
= & E\left[\exp \left\{\sigma W_{T}-\frac{1}{2} \sigma^{2} T\right\} \cdot \exp \left\{(1-E(V)) \int_{0}^{T} \lambda(s) d s\right\} \prod_{i=1}^{n} V_{i} I_{\left\{N_{T}=n\right\}}\right] \\
= & P_{n}(T)(E(V))^{n} \exp \left\{(1-E(V)) \int_{0}^{T} \lambda(s) d s\right\} \\
& E_{*}\left[f\left(V_{1}, V_{2}, \cdots, V_{n}\right)\right]=E\left[f\left(V_{1}, V_{2}, \cdots, V_{n}\right) \frac{d P^{*}}{d P}\right] \\
= & E\left[f\left(V_{1}, V_{2}, \cdots, V_{n}\right) \exp \left\{(1-E(V)) \int_{0}^{T} \lambda(s) d s\right\} \prod_{i=1}^{N_{T}} V_{i}\right] \\
= & E\left[\exp \left\{(1-E(V)) \int_{0}^{T} \lambda(s) d s\right\} \prod_{i=n+1}^{N_{T}} V_{i}\right] E\left[f\left(V_{1}, V_{2}, \cdots, V_{n}\right) \prod_{i=1}^{n} V_{i}\right] \\
= & \frac{1}{(E(V))^{n}} E\left[f\left(V_{1}, V_{2}, \cdots, V_{n}\right) \cdot \prod_{i=1}^{n} V_{i}\right]
\end{aligned}
$$

If the jumps $V_{i}, i \geq 1$ have a lognormal distribution with mean parameter $\mu_{0}$ and variance $\sigma_{0}^{2}$ under the martingale measure $P$, then

$$
\begin{aligned}
& P^{*}\left(\ln V_{i}<x\right)=E^{*}\left(I_{\left\{\ln V_{i}<x\right\}}\right) \\
= & E\left[\exp \left\{\left(1-E(V) \int_{0}^{T} \lambda(s) d s\right\} \prod_{i=1}^{N_{T}} V_{i} I_{\left\{\ln V_{i}<x\right\}}\right]\right. \\
= & \frac{E\left[V_{i} I_{\left\{\ln V_{i}<x\right\}}\right]}{E(V)} \\
= & \exp \left\{-\mu_{0}-\frac{1}{2} \sigma_{0}^{2}\right\} \int_{-\infty}^{x} e^{y} \frac{1}{\sqrt{2 \pi} \sigma_{0}} \exp \left\{-\frac{\left(y-\mu_{0}\right)^{2}}{2 \sigma_{0}^{2}}\right\} d y \\
= & \frac{1}{\sqrt{2 \pi} \sigma_{0}} \int_{-\infty}^{x} \exp \left\{-\frac{\left(y-\left(\mu_{0}+\sigma_{0}^{2}\right)\right)^{2}}{2 \sigma_{0}^{2}}\right\} d y
\end{aligned}
$$

\section{MAIN RESULTS}

Before we want the formula for the value of a Europeantype option. This option is simultaneously a call option on asset $S_{t}^{2}$ with exercise price $S_{t}^{1}$ and a put option on asset $S_{t}^{1}$ with exercise price $_{t}^{S_{t}^{2}}$.

Proposition 1 Assume that the dynamics of a bond $B_{t}$ and two risky assets $S_{t}^{1}, S_{t}^{2}$ are given by (1),(2),(3), respectively. Then the price of a European-type option with a call option on asset $S_{t}^{2}$ with exercise price $S_{t}^{1}$ and expiry date $T$ is given by the expression

$$
\begin{gathered}
C\left(0, S_{T}^{1}, S_{T}^{2}\right)=\sum_{n=0}^{\infty} P_{n}(T) E\left[S_{0}^{2} \exp \left\{(1-E(U)) \int_{0}^{T} \lambda(s) d s\right\} \prod_{i=1}^{n} U_{i} \Phi\left(d_{1}\right)-\right. \\
\left.S_{0}^{1} \exp \left\{(1-E(V)) \int_{0}^{T} \lambda(s) d s\right\} \prod_{i=1}^{n} V_{i} \Phi\left(d_{2}\right)\right] \\
\text { where } \quad d_{1}=\frac{\ln \frac{S_{0}^{2} \prod_{i=1}^{n} \frac{U_{i}}{S_{i}^{1}}}{S_{0}^{1}}+(E(V)-E(U)) \int_{0}^{T} \lambda(s) d s+\frac{1}{2} \sigma^{2} T}{\sigma \sqrt{T}}, \\
d_{2}=d_{1}-\sigma \sqrt{T}, \sigma=\sqrt{\sigma_{1}^{2}-2 \sigma_{1} \sigma_{2} \rho+\sigma_{2}^{2}}
\end{gathered}
$$

Proof Since $P$ is risk-neutral martingale measure,we have

$$
C\left(0, S_{T}^{1}, S_{T}^{2}\right)=E\left[\frac{1}{B_{T}}\left(S_{T}^{2}-S_{T}^{1}\right)^{+}\right]=E\left[\frac{S_{T}^{2}}{B_{T}} I_{\left\{S_{T}^{2} \geq S_{T}^{1}\right\}}\right]-E\left[\frac{S_{T}^{1}}{B_{T}} I_{\left\{S_{T}^{2} \geq S_{T}^{1}\right\}}\right]
$$

For $E\left[\frac{S_{T}^{2}}{B_{T}} I_{\left\{S_{T}^{2} \geq S_{T}^{1}\right\}}\right]$, let $X_{t}=\frac{S_{t}^{1}}{S_{t}^{2}}$, applying Ito's lemma yield ${ }^{[9]}$

$$
\frac{d X_{t}}{X_{t^{-}}}=\left(\sigma_{2}^{2}-\sigma_{1} \sigma_{2} \rho\right) d t+\left(\sigma_{1} \rho-\sigma_{2}\right) d W_{t}^{2}+\sigma_{1} \sqrt{1-\rho^{2}} d W_{t}^{3}+\frac{V-U}{U} d \tilde{N}_{t}
$$

or equivalently

$$
\frac{d X_{t}}{X_{t^{-}}}=\sigma d W_{t}+\frac{V-U}{U} d \tilde{N}_{t}
$$

where $\sigma=\sqrt{\sigma_{1}^{2}-2 \sigma_{1} \sigma_{2} \rho+\sigma_{2}^{2}}, W_{t}=\frac{\sigma_{1} \rho-\sigma_{2}}{\sigma}\left(W_{t}^{2}-\sigma_{2} t\right)+\frac{\sigma_{1} \sqrt{1-\rho^{2}}}{\sigma} W_{t}^{3}$.

Definite by

$$
\frac{d P^{*}}{d P}=\frac{S_{T}^{2}}{S_{0}^{2} B_{T}}=\prod_{i=1}^{N_{T}} U_{i} \exp \left\{-\frac{1}{2} \sigma_{2}^{2} T+\sigma_{2} W_{T}^{2}+(1-E(U)) \int_{0}^{T} \lambda(s) d s\right\}
$$

It is not hard to check that $W_{t}^{3}$ and $W_{t}^{2}-\sigma_{2} t(0 \leq t \leq T)$ follow one-dimensional standard Wiener processes under the martingale measure $P^{*}$, and their mutually independent. Then it 
is easy that $W_{t}=\frac{\sigma_{1} \rho-\sigma_{2}}{\sigma}\left(W_{t}^{2}-\sigma_{2} t\right)+\frac{\sigma_{1} \sqrt{1-\rho^{2}}}{\sigma} W_{t}^{3}$ is standard onedimensional Wiener process under the martingale measure $P^{*}$.

The unique solution of stochastic differential equation (6)equals ${ }^{[10]}$

$$
X_{T}=X_{0} \prod_{i=1}^{N_{T}}\left(\frac{V_{i}}{U_{i}}\right) \exp \left\{-\frac{1}{2} \sigma^{2} T+\sigma W_{T}-E_{*}\left[\frac{V-U}{U}\right] E(U) \int_{0}^{T} \lambda(s) d s\right\}
$$

By Lemma $1 \quad E_{*}\left[\frac{V-U}{U}\right]=E\left[\frac{V-U}{U} \cdot \frac{U}{E(U)}\right]=\frac{E(V)-E(U)}{E(U)}$

(7) equivalently

$$
X_{T}=X_{0} \prod_{i=1}^{N_{T}}\left(\frac{V_{i}}{U_{i}}\right) \exp \left\{-\frac{1}{2} \sigma^{2} T+\sigma W_{T}+(E(U)-E(V)) \int_{0}^{T} \lambda(s) d s\right\}
$$

let $\quad X_{T}^{n}=X_{0} \prod_{i=1}^{n}\left(\frac{V_{i}}{U_{i}}\right) \exp \left\{-\frac{1}{2} \sigma^{2} T+\sigma W_{T}+(E(U)-E(V)) \int_{0}^{T} \lambda(s) d s\right\}$ thus

$$
\begin{aligned}
& E\left[\frac{S_{T}^{2}}{B_{T}} I_{\left\{s_{T}^{2} \geq S_{T}^{1}\right\}}\right]=S_{0}^{2} E_{*}\left[I_{\left\{X_{T} \leq\right\}}\right]=S_{0}^{2} \sum_{n=0}^{\infty} P_{n}^{*}(T) E_{*}\left[P^{*}\left(X_{T}^{n} \leq 1\right)\right] \\
&= S_{0}^{2} \sum_{n=0}^{\infty} P_{n}(T)(E(U))^{n} \exp \left\{(1-E(U)) \int_{0}^{T} \lambda(s) d s\right\} E_{*}\left[P^{*}\left(X_{T}^{n} \leq 1\right)\right] \\
&= S_{0}^{2} \sum_{n=0}^{\infty} P_{n}(T) \exp \left\{(1-E(U)) \int_{0}^{T} \lambda(s) d s\right\} E\left[P^{*}\left(X_{T}^{n} \leq 1\right) \prod_{i=1}^{n} U_{i}\right] \\
& P^{*}\left(X_{T}^{n} \leq 1\right)=P^{*}\left(\ln X_{T}^{n} \leq 0\right) \\
&=P^{*}\left(\ln X_{0} \prod_{i=1}^{n}\left(\frac{V_{i}}{U_{i}}\right) \exp \left\{-\frac{1}{2} \sigma^{2} T+\sigma W_{T}+(E(U)-E(V)) \int_{0}^{T} \lambda(s) d s\right\} \leq 0\right) \\
&=P^{*}\left(\sigma W_{T} \leq \ln \frac{S_{0}^{2} \prod_{i=1}^{n} \frac{U_{i}}{V_{i}^{1}}}{S_{0}^{1}}+(E(V)-E(U)) \int_{0}^{T} \lambda(s) d s+\frac{1}{2} \sigma^{2} T\right) \\
&=P^{*}\left(\sigma W_{T} \leq d\right) \\
&= \Phi\left(\frac{d}{\sigma \sqrt{T}}\right)
\end{aligned}
$$

where $\quad d=\ln \frac{S_{0}^{2} \prod_{i=1}^{n} \frac{U_{i}}{V_{i}}}{S_{0}^{1}}+(E(V)-E(U)) \int_{0}^{T} \lambda(s) d s+\frac{1}{2} \sigma^{2} T$

By virtue of (9)and(10), we have

$E\left[\frac{S_{T}^{2}}{B_{T}} I_{\left\{S_{T}^{2} \geq S_{T}^{1}\right\}}\right]=S_{0}^{2} \sum_{n=0}^{\infty} P_{n}(T) \exp \left\{(1-E(U)) \int_{0}^{T} \lambda(s) d s\right\} E\left[\Phi\left(d_{1}\right) \prod_{i=1}^{n} U_{i}\right]$

where

$$
d_{1}=\frac{d}{\sigma \sqrt{T}}=\frac{\ln \frac{S_{0}^{2} \prod_{i=1}^{n} \frac{U_{i}}{S_{0}^{1}}}{S_{0}^{1}}+(E(V)-E(U)) \int_{0}^{T} \lambda(s) d s+\frac{1}{2} \sigma^{2} T}{\sigma \sqrt{T}}
$$

For $E\left[\frac{S_{T}^{1}}{B_{T}} I_{\left\{S_{T}^{2} \geq S_{T}^{1}\right\}}\right]$, let $Y_{t}=\frac{S_{t}^{2}}{S_{t}^{1}}$

$$
\frac{d P^{*}}{d P}=\frac{S_{T}^{1}}{S_{0}^{1} B_{T}}=\prod_{i=1}^{N_{T}} V_{i} \exp \left\{-\frac{1}{2} \sigma_{1}^{2} T+\sigma_{1} W_{T}^{1}+(1-E(V)) \int_{0}^{T} \lambda(s) d s\right\}
$$

By using the same way we have

$$
E\left[\frac{S_{T}^{1}}{B_{T}} I_{\left\{S_{T}^{2} \geq S_{T}^{1}\right\}}\right]=S_{0}^{1} \sum_{n=0}^{\infty} P_{n}(T) \exp \left\{(1-E(V)) \int_{0}^{T} \lambda(s) d s\right\} E\left[\Phi\left(d_{2}\right) \prod_{i=1}^{n} V_{i}\right]
$$

Where

$$
d_{2}=\frac{d-\sigma^{2} T}{\sigma \sqrt{T}}=\frac{\ln \frac{S_{0}^{2} \prod_{i=1}^{n} \frac{U_{i}}{S_{i}}}{S_{0}^{1}}+(E(V)-E(U)) \int_{0}^{T} \lambda(s) d s-\frac{1}{2} \sigma^{2} T}{\sigma \sqrt{T}}=d_{1}-\sigma \sqrt{T}
$$

Together with(4), (11)and(12),we have proved the following

$$
\begin{aligned}
C\left(0, S_{T}^{1}, S_{T}^{2}\right)= & \sum_{n=0}^{\infty} P_{n}(T) E\left[S_{0}^{2} \exp \left\{(1-E(U)) \int_{0}^{T} \lambda(s) d s\right\} \prod_{i=1}^{n} U_{i} \Phi\left(d_{1}\right)-\right. \\
& \left.S_{0}^{1} \exp \left\{(1-E(V)) \int_{0}^{T} \lambda(s) d s\right\} \prod_{i=1}^{n} V_{i} \Phi\left(d_{2}\right)\right]
\end{aligned}
$$

Proposition 2 Assume that the dynamics of a bond $B_{t}$ and two risky assets $S_{t}^{1}, S_{t}^{2}$ are given by (1),(2),(3), respectively. If $V, U$ have lognormal distribution ${ }^{[11]}$ with mean parameter $\mu_{V}, \mu_{U}$ and variance $\sigma_{V}{ }^{2}, \sigma_{U}{ }^{2}$ under the martingale measure $P$,then the price of a European-type option with a call option on asset $S_{t}^{2}$ with exercise price $S_{t}^{1}$ and expiry date $T$ is given by the expression

$$
\begin{gathered}
C\left(0, S_{T}^{1}, S_{T}^{2}\right)=\sum_{n=0}^{\infty}\left[P_{n}(T) S_{0}^{2} \mu_{U}{ }^{n} \exp \left\{\left(1-\mu_{U}\right) \int_{0}^{T} \lambda(s) d s\right\} \Phi\left(a_{1}\right)-\right. \\
\left.S_{0}^{1} \mu_{V}{ }^{n} \exp \left\{\left(1-\mu_{V}\right) \int_{0}^{T} \lambda(s) d s\right\} \Phi\left(a_{2}\right)\right]
\end{gathered}
$$

where $a_{1}=\frac{\ln \frac{S_{0}^{2}}{S_{0}^{1}}+\left(\mu_{V}-\mu_{U}\right) \int_{0}^{T} \lambda(s) d s+\frac{1}{2} \sigma^{2} T-n\left(\mu_{V}-\mu_{U}-\sigma_{U}{ }^{2}\right)}{\sqrt{\sigma^{2} T+n\left(\sigma_{V}^{2}+\sigma_{U}^{2}\right)}}$,

$$
a_{2}=a_{1}-\sqrt{\sigma^{2} T+{\sigma_{V}}^{2}+{\sigma_{U}}^{2}}, \sigma=\sqrt{\sigma_{1}^{2}-2 \sigma_{1} \sigma_{2} \rho+\sigma_{2}^{2}} .
$$

Proof Using Proposition 1 


$$
\begin{aligned}
C\left(0, S_{T}^{1}, S_{T}^{2}\right) & =\sum_{n=0}^{\infty} P_{n}(T)\left\{S_{0}^{2} \exp \left\{\left(1-\mu_{U}\right) \int_{0}^{T} \lambda(s) d s\right\} E\left[\prod_{i=1}^{n} U_{i} \Phi\left(d_{1}\right)\right]-\right. \\
& \left.S_{0}^{1} \exp \left\{\left(1-\mu_{V}\right) \int_{0}^{T} \lambda(s) d s\right\} E\left[\prod_{i=1}^{n} V_{i} \Phi\left(d_{2}\right)\right]\right\}
\end{aligned}
$$

By using Lemma 1, we have $\sigma W_{t}-\sum_{i=1}^{n} \ln U_{i}+\sum_{i=1}^{n} \ln V_{i}$ would be a normal random variable under the martingale measure $P^{*}$ with mean and variance given by

$$
\begin{aligned}
& E_{*}\left[\sigma W_{t}-\sum_{i=1}^{n} \ln U_{i}+\sum_{i=1}^{n} \ln V_{i}\right]=n\left(\mu_{V}-\mu_{U}-\sigma_{U}{ }^{2}\right), \\
& D_{*}\left[\sigma W_{t}-\sum_{i=1}^{n} \ln U_{i}+\sum_{i=1}^{n} \ln V_{i}\right]=\sigma^{2} t+n\left(\sigma_{V}{ }^{2}+\sigma_{U}{ }^{2}\right)
\end{aligned}
$$

then

$$
\begin{aligned}
& E\left[\prod_{i=1}^{n} U_{i} \Phi\left(d_{1}\right)\right]=\mu_{U}{ }^{n} E_{*}\left[\Phi\left(d_{1}\right)\right] \\
& =\mu_{U}{ }^{n} P^{*}\left(\sigma W_{T} \leq \ln \frac{S_{0}^{2} \prod_{i=1}^{n} \frac{U_{i}}{V_{i}}}{S_{0}^{1}}+(E(V)-E(U)) \int_{0}^{T} \lambda(s) d s+\frac{1}{2} \sigma^{2} T\right) \\
& =\mu_{U}{ }^{n} P^{*}\left(\sigma W_{T}-\sum_{i=1}^{n} \ln U_{i}+\sum_{i=1}^{n} \ln V_{i} \leq a\right) \\
& =\mu_{U}{ }^{n} \Phi\left(\frac{a-n\left(\mu_{V}-\mu_{U}-\sigma_{U}{ }^{2}\right)}{\sqrt{\sigma^{2} T+n\left(\sigma_{V}{ }^{2}+\sigma_{U}{ }^{2}\right.}}\right) \\
& =\mu_{U}{ }^{n} \Phi\left(a_{1}\right)
\end{aligned}
$$

and $\quad E\left[\prod_{i=1}^{n} V_{i} \Phi\left(d_{2}\right)\right]=\mu_{V}{ }^{n} \Phi\left(a_{2}\right)$

where $\quad a=\ln \frac{S_{0}^{2}}{S_{0}^{1}}+\left(\mu_{V}-\mu_{U}\right) \int_{0}^{T} \lambda(s) d s+\frac{1}{2} \sigma^{2} T$

Hence, we have

$$
\begin{gathered}
C\left(0, S_{T}^{1}, S_{T}^{2}\right)=\sum_{n=0}^{\infty}\left[P_{n}(T) S_{0}^{2} \mu_{U}{ }^{n} \exp \left\{\left(1-\mu_{U}\right) \int_{0}^{T} \lambda(s) d s\right\} \Phi\left(a_{1}\right)-\right. \\
\left.S_{0}^{1} \mu_{V}{ }^{n} \exp \left\{\left(1-\mu_{V}\right) \int_{0}^{T} \lambda(s) d s\right\} \Phi\left(a_{2}\right)\right]
\end{gathered}
$$

Proposition 3 Assume that the dynamics of a bond $B_{t}$ and two risky assets $S_{t}^{1}, S_{t}^{2}$ are given by (1),(2),(3), respectively. Then the put-call parity relation may be rewritten as

$$
C\left(t, S_{T}^{1}, S_{T}^{2}\right)-P\left(t, S_{T}^{1}, S_{T}^{2}\right)=S_{t}^{2}-S_{t}^{1}
$$

We can using put-call parity ${ }^{[12]}$ to find the price of a European put option on a stock with the same parameters as earlier.

\section{CONCLUDING REMARKS}

In this paper, we develop an equation for the value of the option to exchange one risky asset for another. Establish the option-pricing model when exercise price is random variable. The option-pricing model is options to exchange one asset to another. Because jumps do occur in practice, it is advantageous to consider a model for price evolution that superimposes random jumps on a geometric Brownian motion. By changing basic assumption of William Margrabe exchange option pricing model to the assumption that jump process is count process that more general than Poisson process, it is established that the behavior model of the stock pricing process is jump-diffusion process. Considering when the jump distribution is lognormal, we get European exchange call and put option pricing formula and their parity.

\section{ACKNOWLEDGMENT}

This work is supported by National Natural Science Foundation of China under Grant No. 71103143,No. 71473194 Science and Technology Foundation of Shaanxi Province of China under Grant No. 2013XJXX-40; Natural Science Foundation of Shaanxi Province of China under Grant No. 2017JQ1486; Scientific Research Program Funded by Shaanxi Provincial Education Department (Program No. 16JK1500).

\section{REFERENCES}

[1] F. Black, M. Scholes, "The pricing of options and corparately abilities," Journal of Political, vol. 81, pp. 637-659, 1973.

[2] W. Margarbe, "The value of an Option to Exchange One Asset for Another," Journal of Finance, vol. 33, pp. 177-186, 1978.

[3] R.C. Merton, "Option pricing when underling process of stock returns is discontinuous," Journal of Financial Economics, vol. 23, pp. 124-144, 1976.

[4] D. Duffie, Dynamic Asset Pricing Theoty. Princeton University Press. New Jersay, 1996.

[5] D. Lamberton, B. Lapeyre, Introduciton to Stochatic Calulus Applied to Finance. Chapman \& Hall, London, 1996.

[6] M. Musiela, M. Rutkowski, Martingale Methods in Financial Modeling. Springer. New York, 1998.

[7] P. Bremaud, Point Processes and Queues: Martingale Dynamic. Springer. New York, 1981

[8] I. Karatzas, S.E. Shreve, Methods of Mathematical Finance. Springer. New York, 1998

[9] P. Protter, Stochastic Integration and Differential Equations. Springer. New York, 1990.

[10] I. Bardhan, X. Chao, Stochastic multi-agent equilibrium in economies with jump-diffusion uncertainty,” Journal of Economics Dynamics and Control, vol. 20, pp. 361-384, 1996.

[11] S. M. Sheldon, An Elementary Introduction to Mathematical Finance: Options and Other Topics. Cambridge University Press, 2003.

[12] F. Stamofli, V. Goodma, The Mathematics of Fianace: Modeling and Hedging. Thomson Learning, 2001. 